\title{
$D$-wave charmonia
}

\author{
Dian-Yong Chen, ${ }^{1, *}$ Cheng-Qun Pang, ${ }^{2, \uparrow}$ Jun He, ${ }^{3, *}$ and Zhi-Yong Zhou ${ }^{1, \S}$ \\ ${ }^{1}$ School of Physics, Southeast University, Nanjing 210094, People's Republic of China \\ ${ }^{2}$ College of Physics and Electronic Information, Qinghai Normal University, \\ Xining 810000, People's Republic of China \\ ${ }^{3}$ Department of Physics and Institute of Theoretical Physics, Nanjing Normal University, \\ Nanjing 210097, People's Republic of China
}

(Received 14 August 2019; published 15 October 2019)

\begin{abstract}
Inspired by the recent observations of the vector charmoniumlike states at BES III Collaboration and $\psi(3842)$ at LHCb Collaboration, we comb the $D$ wave charmonium state in the present work. We first evaluate the possibility of $Y(4320)$ as $\psi\left(3^{3} D_{1}\right)$ by investigating its open charm decays in the quark-pair creation model and we find the width of $Y(4320)$ can be reproduced in a reasonable parameter range. Moreover, we take $\psi(3770), \psi(4160)$, and $Y(4320)$ as the scale of $1 D, 2 D$, and $3 D$ charmonia to estimate the open charm decays of other $D$ wave charmonia. The total and partial widths of $D$ wave charmonium states have been predicted, which could be tested by further measurements at LHCb and Belle II Collaborations.
\end{abstract}

DOI: 10.1103/PhysRevD.100.074016

\section{INTRODUCTION}

By analyzing the precise cross sections for $e^{+} e^{-} \rightarrow \omega \chi_{c 0}$ [1], $e^{+} e^{-} \rightarrow \pi^{+} \pi^{-} J / \psi$ [2], $e^{+} e^{-} \rightarrow \pi^{+} \pi^{-} h_{c}$ [3], and $e^{+} e^{-} \rightarrow \pi^{+} D^{0} D^{*-}$ [4], the BESIII Collaboration reported a series of vector charmoniumlike states, which are $Y(4220), Y(4320)$, and $Y(4390)$. The charmoniumlike state $Y(4220)$ has been reported in $\chi_{c 0} \omega, \pi^{+} D^{0} D^{*-}$, $\pi^{+} \pi^{-} h_{c}$, and $\pi^{+} \pi^{-} J / \psi$ channels at present. Its width were reported to be around $40 \mathrm{MeV}$ by analyzing the cross sections for $e^{+} e^{-} \rightarrow \chi_{c 0} \omega$ and $e^{+} e^{-} \rightarrow \pi^{+} \pi^{-} J / \psi$, while it were measured to be about $70 \mathrm{MeV}$ in the cross sections for $e^{+} e^{-} \rightarrow \pi^{+} \pi^{-} h_{c}$ process. As for $Y(4320)$, it was a broad charmoniumlike state and only reported in $\pi^{+} \pi^{-} J / \psi$ process. The charmoniumlike state $Y(4390)$ is also a broad state and was observed in the spin flipped $\pi^{+} \pi^{-} h_{c}$ channel.

These newly observed charmoniumlike states make resonances with $J^{P C}=1^{--}$between $4.0-4.5 \mathrm{GeV}$ overcrowded and the nature of these charmoniumlike states becomes an intriguing question. As for $Y(4220)$, it has been

\footnotetext{
*Corresponding author. chendy@seu.edu.cn

Corresponding author.

pcq@qhnu.edu.cn

junhe@njnu.edu.cn

§zhouzhy@seu.edu.cn

Published by the American Physical Society under the terms of the Creative Commons Attribution 4.0 International license. Further distribution of this work must maintain attribution to the author(s) and the published article's title, journal citation, and DOI. Funded by SCOAP ${ }^{3}$.
}

observed in various channels. In the $\pi^{+} \pi^{-} J / \psi$ channel, a structure, $Y(4260)$ was first reported by the $B A B A R$ Collaboration [5] and then confirmed by the Belle Collaboration [6]. Recent precise analysis from BESIII Collaboration indicates the structure $Y(4260)$ should contain two charmoniumlike state, $Y(4220)$ and $Y(4320)$ [2]. The former one is consistent with the one observed in the channels of $\chi_{c 0} \omega, \pi^{+} \pi^{-} h_{c}$ and $\pi^{+} D^{0} D^{*-}$. Since $Y(4260) /$ $Y(4220)$ is close to $D_{1}(2420) \bar{D}$ threshold, it could be considered as a molecular state composed of $D_{1}(2420) \bar{D}^{1}$ [7-12]. While, the QCD sum rule estimations indicate that $Y(4260)$ could be a mixed charmonium-tetraquark state $[13,14]$.

Before the observations of $Y(4220)$, we predicted a narrow $\psi(4 S)$ around $4.2 \mathrm{GeV}$ in Ref. [15], while $\psi(4415)$ was considered as $\psi(5 S)$. After the observation of $Y(4220)$ in the $\chi_{c 0} \omega$ channel, The possibility of $Y(4220)$ as $\psi(4 S)$ was further evaluated $[16,17]$. As for $Y(4390)$, it is only observed in the $\pi^{+} \pi^{-} h_{c}$ channel. In Refs. [18,19], the possibility of $Y(4390)$ as a $D^{*} D_{1}(2420)$ molecular state were investigated. While in Ref. [20], the lineshapes of the cross sections for $e^{+} e^{-} \rightarrow \pi^{+} \pi^{-} J / \psi, \pi^{+} \pi^{-} h_{c}, \pi^{+} D^{-} D^{*-}$ could be well reproduced by interferences of the well established charmonia $\psi(4160)$ and $\psi(4415)$ as well as $Y(4220)$.

As for $Y(4320)$, it was also observed in the $\pi^{+} \pi^{-} J / \psi$ channel firstly. Actually, in the $\pi^{+} \pi^{-} \psi(2 S)$ channel, there exists a charmoniumlike state $Y(4360)$ near the newly observed $Y(4320)$ [21,22]. The mass of $Y(4360)$ was fitted

\footnotetext{
${ }^{1}$ The charge conjugate states are implied throughout this work.
} 
to be $4324 \pm 24 \mathrm{MeV}$ by BABAR Collaboration [21], which is consistent with the mass of $Y(4320)$. In addition, with recent precise data, the analysis in Ref. [23] also indicates that the charmoniumlike states $Y(4360)$ in the $\pi^{+} \pi^{-} \psi(2 S)$ channel and $Y(4320)$ in the $\pi^{+} \pi^{-} J / \psi$ channel should be the same state.

In our previous work, we have categorized $Y(4220)$ as $\psi(4 S)$ and $\psi(4415)$ as $\psi(5 S)$ [15-17]. In such a scenario, there are no additional room left for $Y(4320)$ in the $S$-wave vector charmonium and in the vicinity of $Y(4320)$, there is no charmed mesons pair threshold. However, if one further checks the charmonium spectroscopy, one can find that in the $D$-wave charmonium sector, $\psi(3770)$ and $\psi(4160)$ are well established as $\psi\left(1^{3} D_{1}\right)$ and $\psi\left(2^{3} D_{1}\right)$ states, respectively. The higher $D$-wave vector charmonia have not been observed experimentally. On the theoretical side, the masses of $D$ wave charmonia have been predicted in the quark model as shown in Table I. One can find the mass of $\psi\left(3^{3} D_{1}\right)$ was predicted to be $4519 \mathrm{MeV}$ by the relativistic quark model [24]. However, for the higher charmonia, the couple channel effects will shift their mass to the open-charm threshold [25-27], thus the predicted mass of $\psi\left(3^{3} D_{1}\right)$ in Ref. [24] should be too large since the coupled-channel effects are not included. In Refs. [28,29], the screened potential model were employed to depict the couple channel effect in the charmonium, the predicted mass of $\psi\left(3^{3} D_{1}\right)$ is $4317 \mathrm{MeV}$ and $4334 \mathrm{MeV}$, respectively, which is well consistent with the one of $Y(4320)$. Thus, $Y(4320)$ could be a good candidate of $\psi\left(3^{3} D_{1}\right)$ state.

Moreover, very recently, the LHCb collaboration reported their measurements of the near threshold $D \bar{D}$ spectroscopy [31]. In the $D \bar{D}$ mass spectrum, the $D$ wave charmonium $\psi(3770)$ was observed in the hadronproduction process for the first time [31]. In the same spectroscopy, a new narrow state (named $\psi(3842)$ hereafter) was reported. As shown in Table I, the mass of this newly observed state is consistent with one of $\psi\left(1^{3} D_{3}\right)$ state predicted by quark model $[24,28,29]$ and the narrow width could result from the higher partial wave suppression since $\psi\left(1^{3} D_{3}\right)$ decays into $D \bar{D}$ via a $F$ wave with $L=3$. Moreover, another $D$-wave charmonia candidate, $\psi(3823)$, was firstly observed by Belle Collaboration [32] and then confirmed by BES III Collaboration [33]. Considering $\psi(3823)$ as $\psi_{2}(1 D)$ state, together with the newly observed $\psi(3842)$ as $\psi_{3}(1 D)$ state, the $D$ wave ground spin triplets have been well established. As for $2 D$ charmonia, one can find only $\psi_{1}(2 D)$ state has been observed experimentally. Thus, searching the missing highly excited $D$ wave charmonia experimentally will be intriguing. Unlike to the electron-positron annihilation process, the states produced in the hadronproduction process have more possibility of $J^{P C}$ quantum numbers, while states involved in the electron-positron annihilation process have fixed $J^{P C}$ quantum numbers, which are $1^{--}$. Thus, the hadronproduction process in the $\mathrm{LHCb}$ Collaboration provide us a powerful platform of searching for charmonium states with various $J^{P C}$ quantum numbers, which includes the missing highly excited $D$ wave charmonia.

On the theoretical side, it will be intriguing to comb the $D$ wave charmonium states. In the present work, we take $\psi(3770), \psi(4160)$ and $Y(4320)$ as the $\psi\left(1^{3} D_{1}\right), \psi\left(2^{3} D_{1}\right)$ and $\psi\left(3^{3} D_{1}\right)$ charmonia, and take these states as scales to investigate the open charm decays of other $D$ wave charmonium states, which could, to some extend, cancel the uncertainties of quark model.

This work is organized as follows. After the Introduction, a short review of the quark pair creation model and the formula of open-charm decays of $D$ wave charmonium states are presented in Sec. II. Our numerical results and discussions are given in Sec. III. Section IV is devoted to summary.

TABLE I. Mass spectra and $R$ values of $D$-wave charmonia. SP, GI and MGI refer to the screen potential model [28], Godfrey-Isgur relativistic quark model [24] and modified Godfrey-Isgur relativistic quark model [29]. The values in the bracket are the effective $R$ values of the corresponding states in unit of $\mathrm{GeV}^{-1}$.

\begin{tabular}{lcccc}
\hline \hline States & Experiment & SP Model [28] & GI Model [24] & MGI Model [29] \\
\hline$\eta_{c 2}(1 D)$ & $\ldots$ & 3796 & 3837 & 3848 \\
$\psi_{1}(1 D)$ & $3773.13 \pm 0.15[30]$ & $3783(2.59)$ & $3821(1.84)$ & $3830(1.88)$ \\
$\psi_{2}(1 D)$ & $3822.2 \pm 1.2[30]$ & 3798 & 3838 & 3848 \\
$\psi_{3}(1 D)$ & $3842.71 \pm 1.6 \pm 0.12[31]$ & 3799 & 3846 & 3858 \\
$\eta_{c 2}(2 D)$ & $\ldots$ & 4099 & 4207 & 4137 \\
$\psi_{1}(2 D)$ & $4191 \pm 5[30]$ & $4089(3.12)$ & $4197(2.09)$ & $4125(2.38)$ \\
$\psi_{2}(2 D)$ & $\ldots$ & 4100 & 4209 & 4137 \\
$\psi_{3}(2 D)$ & $\ldots$ & 4103 & 4215 & 4144 \\
$\eta_{c 2}(3 D)$ & $\ldots$ & 4326 & 4531 & 4343 \\
$\psi_{1}(3 D)$ & $\ldots$ & $4317(3.59)$ & $4522(2.24)$ & $433(2.85)$ \\
$\psi_{2}(3 D)$ & $\ldots$ & 4327 & 4532 & 4343 \\
$\psi_{3}(3 D)$ & $\ldots$ & 4331 & 4536 & 4348 \\
\hline \hline
\end{tabular}




\section{QUARK PAIR CREATION MODEL AND OPEN CHARM DECAYS OF $\psi\left({ }^{3} D_{1}\right)$ CHARMONIUM}

\section{A. Review of quark pair creation model}

Here, we adopt the quark pair creation (QPC) model (also named ${ }^{3} P_{0}$ model since the $J^{P C}$ quantum numbers of the quark pair created from the vacuum are $0^{++}$) to estimate the open charm decays of charmonia. The QPC model was first proposed by Micu [34-37] and then widely used to estimate the Okubo-Zweig-lizuka allowed strong decay processes [29,38-45]. In the QPC model, the related $S$ matrix of $A \rightarrow B C$ process reads,

$$
\langle B C|S| A\rangle=I-i 2 \pi \delta\left(E_{f}-E_{i}\right)\langle B C|\mathcal{T}| A\rangle,
$$

where the transition operator $\mathcal{T}$ is,

$$
\begin{aligned}
\mathcal{T}= & -3 \gamma \sum_{m}\langle 1 m ; 1-m \mid 00\rangle \int d \mathbf{k}_{3} \mathbf{k}_{4} \delta^{3}\left(\mathbf{k}_{3}+\mathbf{k}_{4}\right) \\
& \times \mathcal{Y}_{1 m}\left(\frac{\mathbf{k}_{3}-\mathbf{k}_{4}}{2}\right) \chi_{1,-m}^{34} \varphi_{0}^{34} \omega_{0}^{34} d_{3 i}^{\dagger}\left(\mathbf{k}_{3}\right) b_{4 j}^{\dagger}\left(\mathbf{k}_{4}\right),
\end{aligned}
$$

where $\mathcal{Y}_{1 m}(\mathbf{k})=|\mathbf{k}| Y_{1 m}(\theta, \phi), \chi_{1,-m}^{34}, \varphi_{0}^{34}=(u \bar{u}+d \bar{d}+$ $s \bar{s}) / \sqrt{3}$ and $\omega_{0}^{34}=\delta_{\alpha_{3} \alpha_{4}}$ are the space, spin, flavor, and color parts of the wave functions, respectively. $\alpha_{3}$ and $\alpha_{4}$ are the color indexes of the created quark pair. In the QPC model, the parameter $\gamma$ is introduced to represent the strength of the quark-antiquark pair creation from the vacuum and it could be fixed by fitting the decay data. In the present work, we take $\gamma=6.3$ for the up/down quark pair and $\gamma_{s}=\gamma / \sqrt{3}$ for strange quark pair creation [38,39].

In the initial rest frame, the matrix element of the transition operator is

$$
\begin{aligned}
& \langle B C|\mathcal{T}| A\rangle \\
& =\sqrt{8 E_{A} E_{B} E_{C}} \gamma \sum_{\substack{M_{L_{A}}, M_{L_{B}}, M_{L_{C}} \\
M_{S_{A}}, M_{B}, M_{S_{C}}}}\langle 1 m, 1-m \mid 00\rangle \\
& \quad \times\left\langle L_{A}, M_{L_{A}}, S_{A} M_{S_{A}} \mid J_{A}, M_{A}\right\rangle\left\langle L_{B}, M_{L_{B}}, S_{B} M_{S_{B}} \mid J_{B}, M_{B}\right\rangle \\
& \quad \times\left\langle L_{C}, M_{L_{C}}, S_{C} M_{S_{C}} \mid J_{C}, M_{C}\right\rangle\left\langle\varphi_{B}^{13} \varphi_{C}^{24} \mid \varphi_{A}^{12} \varphi_{0}^{34}\right\rangle \\
& \quad \times\left\langle\chi_{S_{B} M_{S_{B}}}^{13} \chi_{S_{C} M_{S_{C}}^{24}}^{24} \chi_{S_{A} M_{S_{A}}^{12}}^{12} \chi_{1-m}^{34}\right\rangle I_{M_{L_{B}} M_{L_{C}}}^{M_{L_{A}} m}(\mathbf{K}),
\end{aligned}
$$

where $\left\langle\varphi_{B}^{13} \varphi_{C}^{24} \mid \varphi_{A}^{12} \varphi_{0}^{34}\right\rangle$ and $\left\langle\chi_{S_{B} M_{S_{B}}}^{13} \chi_{S_{C} M_{S_{C}}}^{24} \mid \chi_{S_{A} M_{S_{A}}}^{12} \chi_{1-m}^{34}\right\rangle$ are the flavor matrix element and spin matrix element, respectively. While the color matrix element $\left\langle\omega_{B}^{13} \omega_{C}^{24} \mid \omega_{A}^{12} \omega_{0}^{34}\right\rangle=$ $1 / 3$ cancels out the factor 3 in the transition operator defined in Eq. (2). The matrix element of the spatial part reads

$$
\begin{aligned}
I_{M_{L_{B}}, M_{L_{C}}}^{M_{L_{A}}, m}(\mathbf{K})= & \int d \mathbf{k}_{1} \mathrm{~d} \mathbf{k}_{2} \mathrm{~d} \mathbf{k}_{3} \mathrm{~d} \mathbf{k}_{4} \delta^{3}\left(\mathbf{k}_{1}+\mathbf{k}_{2}\right) \delta^{3}\left(\mathbf{k}_{3}+\mathbf{k}_{4}\right) \\
& \times \delta^{3}\left(\mathbf{K}_{B}-\mathbf{k}_{1}-\mathbf{k}_{3}\right) \delta^{3}\left(\mathbf{K}_{C}-\mathbf{k}_{2}-\mathbf{k}_{4}\right) \\
& \times \Psi_{n_{B} L_{B} M_{L_{B}}}^{*}\left(\mathbf{k}_{1}, \mathbf{k}_{3}\right) \Psi_{n_{C} L_{C} M_{L_{C}}}^{*}\left(\mathbf{k}_{2}, \mathbf{k}_{4}\right) \\
& \times \Psi_{n_{A} L_{A} M_{L_{A}}}\left(\mathbf{k}_{1}, \mathbf{k}_{2}\right) \mathcal{Y}_{1 m}\left(\frac{\mathbf{k}_{3}-\mathbf{k}_{4}}{2}\right)
\end{aligned}
$$

which reflects the overlap of the spatial wave functions of the initial state and final states. The amplitude of the decay process is

$$
\langle B C|\mathcal{T}| A\rangle=\delta^{3}\left(\mathbf{K}_{B}+\mathbf{K}_{C}-\mathbf{K}_{A}\right) \mathcal{M}^{M_{J_{A}} M_{J_{B}} M_{J_{C}}} .
$$

By the Jacobi-Wick rotation, the amplitude can be transformed into partial wave amplitude, which is,

$$
\begin{aligned}
\mathcal{M}^{J L}(A \rightarrow B C)= & \frac{\sqrt{2 L+1}}{2 J_{A}+1} \sum_{M_{J_{B}}, M_{J_{C}}}\left\langle L 0 J M_{J_{A}} \mid J_{A} M_{J_{A}}\right\rangle \\
& \times\left\langle J_{B} M_{J_{B}} J_{C} M_{J_{C}} \mid J_{A} M_{J_{A}}\right\rangle \mathcal{M}^{M_{J_{A}} M_{J_{B}} M_{J_{C}}} .
\end{aligned}
$$

In terms of the partial wave amplitude, the partial width is

$$
\Gamma=\pi^{2} \frac{|\mathbf{K}|}{m_{A}^{2}} \sum_{J L}\left|\mathcal{M}^{J L}\right|
$$

where $|\mathbf{K}|=\lambda^{1 / 2}\left(m_{A}^{2}, m_{B}^{2}, m_{C}^{2}\right)$ with the Källen function $\lambda(x, y, z)=x^{2}+y^{2}+z^{2}-2 x y-2 y z-2 x z$.

\section{B. Open charm decays of $\boldsymbol{D}$-wave charmonia}

In the present work, we perform a system estimation of the open charm decays of $D$ wave charmonia. To evasion the uncertainties of quark model, we take $\psi(3770)$, $\psi(4160)$. and $Y(4320)$ as the scale of $1 D, 2 D$, and $3 D$ charmonium states. The masses of the involved charmonium states and charmed mesons are listed in Table II. As for the charmed mesons and already established charmonia, i.e., $\psi(3770)$ and $\psi(4160)$, we adopt the center values of the particle data group (PDG) average [30]. As for $Y(4320)$ and $\psi_{3}(3842)$, we take the measurement one in Ref. [2,31]. It is interesting to notice the mass splitting between the same spin multiplets are predicted to be very similar for different quark models $[24,28,29]$. For example, the mass splitting $\Delta m_{a}=$ $m_{\eta_{c 2}(2 D)}-m_{\psi_{1}(2 D)}$ are predicted to 10,12 , and $10 \mathrm{MeV}$ for Godfrey-Isgur (GI) model, Modied-Godfrey-Isgur (MGI) model, and screen potential (SP) model, respectively. By using the mass splitting estimated in the SP model and taking $\psi(4160)$ as the scale of $2 D$ states, the masses of the missing $2 D$ states can be estimated, e.g., $m_{\eta_{c 2}(2 D)}=$ $m_{\psi(4160)}+\Delta m_{a}=4201 \mathrm{MeV}$. In the same way, the masses 
TABLE II. The masses and $R$ values of the involved mesons. Here $( \pm)$ and $(0)$ indicate the charge of the mesons.

\begin{tabular}{lcc}
\hline \hline Meson & Mass $(\mathrm{MeV})$ & $R\left(\mathrm{GeV}^{-1}\right)[46]$ \\
\hline$D$ & $1864.83(0), 1869.58( \pm)$ & 1.52 \\
$D^{*}$ & $2006.85(0), 2010.26( \pm)$ & 1.85 \\
$D_{0}(2400)$ & $2318(0) 2351( \pm)$ & 1.85 \\
$D_{1}(2420)$ & $2420.8(0), 2423.2( \pm)$ & 2.00 \\
$D_{1}^{\prime}(2430)$ & $2427(0), 2427( \pm)$ & 2.00 \\
$D_{2}(2460)$ & $2460.7(0), 2465.4( \pm)$ & 2.00 \\
$D_{s}$ & $1968.28( \pm)$ & 1.41 \\
$D_{s}^{*}$ & $2112.1( \pm)$ & 1.69 \\
$\psi(3770)$ & 3773.13 & $\ldots$ \\
$\psi_{3}(3842)$ & 3842.71 & $\ldots$ \\
$\eta_{c 2}(2 D)$ & 4201 & $\ldots$ \\
$\psi(4160)$ & 4191 & $\ldots$ \\
$\psi_{2}(2 D)$ & 4203 & $\ldots$ \\
$\psi_{3}(2 D)$ & 4209 & $\ldots$ \\
$\eta_{c 2}(3 D)$ & 4330 & $\ldots$ \\
$Y(4320)$ & 4320.0 & $\ldots$ \\
$\psi_{2}(3 D)$ & 4330 & $\ldots$ \\
$\psi_{3}(3 D)$ & 4335 & \\
\hline \hline
\end{tabular}

of the missing $3 D$ states can be evaluated by taking $Y(4320)$ as the scale.

Considering the $J^{P C}$ conservation and kinetics limit, we list all the possible open charm decay modes of $D$ wave charmonia in Table III. As for ground states, $\psi_{1}(1 D)$ and $\psi_{3}(1 D)$ can decay into $D \bar{D}$ via $P$ wave and $F$ wave, respectively, while $\eta_{c 2}(1 D)$ and $\psi_{2}(1 D)$ have no open charm decay mode, although they are above the threshold of $D \bar{D}$. With Eqs. (1)-(6), one can get the partial wave amplitudes of the involved process as shown in Table III. The estimated particular expressions of these partial wave amplitudes are listed in Table IV-VII in the Appendix.

For $3 D$ charmonia, their masses are above the threshold of $D_{1}^{\prime}(2430) \bar{D}$ and $D_{1}(2420) \bar{D}$. The charmed meson $D_{1}^{\prime}(2430)$ and $D_{1}(2420)$ are the mixture of the $1^{3} P_{1}$ and $1^{1} P_{1}$ states and the mixing scheme is,

$$
\left(\begin{array}{c}
\left|D_{1}^{\prime}(2430)\right\rangle \\
\left|D_{1}(2420)\right\rangle
\end{array}\right)=\left(\begin{array}{cc}
\cos \theta & \sin \theta \\
-\sin \theta & \cos \theta
\end{array}\right)\left(\begin{array}{l}
\left|1^{1} P_{1}\right\rangle \\
\left|1^{3} P_{1}\right\rangle
\end{array}\right)
$$

where the mixing angle $\theta=-54.7^{\circ}$, which is determined by the heavy quark limit [46-48].

\section{NUMERICAL RESULTS AND DISCUSSIONS}

With the above preparations, we could investigate the open charm decays of the $D$ wave charmonia. In Eq. (4), the spatial wave functions of the mesons are involved. In principle, these wave functions could be estimated by the constitute quark model. However, as we discussed in the introduction, there exist some uncertainties in the quark models. Thus, in the present work, we employ the simple harmonic oscillator wave function to simulate the spatial distribution of the quark-antiquark in meson. The detailed form of the spatial wave function in the momentum representation is

$$
\begin{aligned}
& \Psi_{n \ell m_{\ell}}(R, \mathbf{k}) \\
& =\frac{(-1)^{n}(-i)^{\ell} R^{3 / 2}}{\sqrt{4} \pi} \sqrt{\frac{2^{\ell-n+2}(2 \ell+2 n+1) ! !}{n !(2 \ell+1) ! !^{2}}}(k R)^{\ell} \\
& \quad \times F\left(-n, \ell+\frac{3}{2}, R^{2} k^{2}\right) e^{-R^{2} k^{2} / 2} Y_{\ell, m_{\ell}}(\hat{\mathbf{k}})
\end{aligned}
$$

where $n, \ell$, and $m_{\ell}$ are the radial, angular momentum, and magnetic quantum numbers, respectively. $F(-n, \nu, x)$ and $Y_{\ell m_{\ell}}$ indicate the hypergeometric function and spherical harmonic function, respectively.

In the spatial wave function, a parameter $R$ is introduced. As for the lowest charmed mesons, the predictions of the relativistic quark model are well consistent with the experimental measurements. Thus, in the present work, the values of parameter $R$ for the charmed and charmedstrange mesons are fixed such that it reproduces the root

\begin{tabular}{|c|c|c|c|c|c|c|c|c|c|c|c|c|}
\hline Channel & $\eta_{c 2}(1 D)$ & $\psi_{1}(1 D)$ & $\psi_{2}(1 D)$ & $\psi_{3}(1 D)$ & $\eta_{c 2}(2 D)$ & $\psi_{1}(2 D)$ & $\psi_{2}(2 D)$ & $\psi_{3}(2 D)$ & $\eta_{c 2}(3 D)$ & $\psi_{1}(3 D)$ & $\psi_{2}(3 D)$ & $\psi_{3}(3 D)$ \\
\hline$D \bar{D}$ & $\ldots$ & $\checkmark$ & $\ldots$ & $\checkmark$ & $\ldots$ & $\checkmark$ & $\ldots$ & $\checkmark$ & $\ldots$ & $\checkmark$ & $\ldots$ & $\checkmark$ \\
\hline$D \bar{D}^{*}$ & $\ldots$ & $\ldots$ & $\ldots$ & $\ldots$ & $\checkmark$ & $\checkmark$ & $\checkmark$ & $\checkmark$ & $\checkmark$ & $\checkmark$ & $\checkmark$ & $\checkmark$ \\
\hline$D^{*} \bar{D}^{*}$ & $\ldots$ & $\ldots$ & $\ldots$ & $\ldots$ & $\checkmark$ & $\checkmark$ & $\checkmark$ & $\checkmark$ & $\checkmark$ & $\checkmark$ & $\checkmark$ & $\checkmark$ \\
\hline$D_{s}^{+} D_{s}^{-}$ & $\ldots$ & $\ldots$ & $\ldots$ & $\ldots$ & & $\checkmark$ & $\ldots$ & $\checkmark$ & & $\checkmark$ & $\ldots$ & $\checkmark$ \\
\hline$D_{s}^{+} D_{s}^{*-}$ & $\ldots$ & $\ldots$ & $\ldots$ & $\ldots$ & $\checkmark$ & $\checkmark$ & $\checkmark$ & $\checkmark$ & $\checkmark$ & $\checkmark$ & $\checkmark$ & $\checkmark$ \\
\hline$D_{s}^{*+} D_{s}^{*-}$ & $\ldots$ & $\ldots$ & $\ldots$ & $\ldots$ & 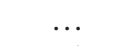 & $\ldots$ & $\ldots$ & $\ldots$ & $\checkmark$ & $\checkmark$ & $\checkmark$ & $\checkmark$ \\
\hline$D \bar{D}_{0}$ & $\ldots$ & $\ldots$ & $\ldots$ & $\ldots$ & $\checkmark$ & $\ldots$ & $\checkmark$ & $\ldots$ & $\checkmark$ & & $\checkmark$ & \\
\hline$D \bar{D}_{1}(2420)$ & $\ldots$ & $\ldots$ & $\ldots$ & $\ldots$ & $\ldots$ & $\ldots$ & $\ldots$ & $\ldots$ & $\checkmark$ & $\checkmark$ & $\checkmark$ & $\checkmark$ \\
\hline$D \bar{D}_{1}(2430)$ & $\ldots$ & $\ldots$ & $\ldots$ & $\ldots$ & $\ldots$ & $\ldots$ & $\ldots$ & $\ldots$ & $\checkmark$ & $\checkmark$ & $\checkmark$ & $\checkmark$ \\
\hline$D^{*} \overline{\bar{D}}_{0}(2400)$ & $\ldots$ & $\ldots$ & $\ldots$ & $\ldots$ & $\ldots$ & $\ldots$ & $\ldots$ & $\ldots$ & $\checkmark$ & $\ldots$ & $\checkmark$ & $\checkmark$ \\
\hline$D_{s}^{+} D_{s 0}^{-}(2317)$ & $\ldots$ & $\ldots$ & $\ldots$ & $\ldots$ & 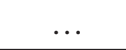 & & $\ldots$ & 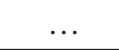 & $\checkmark$ & 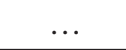 & $\checkmark$ & \\
\hline
\end{tabular}
mean square radius estimated by the relativistic quark model [46]. In Refs. [39,42-45], the simple harmonic

TABLE III. The open charm decay modes of $D$ wave charmonia. 
oscillator wave function with a parameter $R$ has been used to investigate the decay behavior of mesons and the estimated results could well reproduce the corresponding experimental data, which proves such an approach is reliable to investigate the strong decays of the hadrons. As for the charmonia, the $R$ values are quite different in different quark model as shown in Table I. For example, the $R$ value of $1 D$ states are estimated to be $2.59,1.84$ and $1.88 \mathrm{GeV}^{-1}$ by SP, GI and MGI model, respectively. It should be noticed that the mass of charm quark is taken as $1.4045 \mathrm{GeV}$ and $1.65 \mathrm{GeV}$ in the SP and MGI models, respectively. The mass spectra and the wave function depend on both the quark mass and the potential between quark and anti-quark. Thus, the large discrepancy of $R$ values in SP and MGI model could be understood. In the ${ }^{3} P_{0}$ model, the constituent quark masses for the charm, up/ down, strange quarks are adopted to be $1.60,0.22$, and $0.419 \mathrm{GeV}$, respectively [38,39]. The mass of the charm quark in the ${ }^{3} P_{0}$ model is very close to the one in the MGI model. Thus in the present work, we vary the $R$ values of the charmonia around the one of MGI model to check the $R$ dependence of the decay widths. In addition, similar to the case of determining the masses of the missing $D$ wave states, the $R$ values of $1 D, 2 D$, and $3 D$ states could be determined such that it could reproduce the widths of $\psi(3770), \psi(4160)$, and $Y(4320)$, respectively, which could also reduce the uncertainties of quark model. The masses and $R$ values of the involved mesons are presented in Table II.

\section{A. Open charm decays of $1 D$ states}

As for $1 D$ charmonia, their masses are all above the $D \bar{D}$ threshold, however, $\eta_{c 2}$ and $\psi_{2}(1 D)$ cannot decay into $D \bar{D}$ due to $J^{P}$ quantum numbers violation. As for the $\psi(3770)$, the only open charm decay mode is $D \bar{D}$ due to kinematics limit. The $R$ dependence of the partial width of $\psi(3770) \rightarrow$ $D \bar{D}$ is presented in the left panel of Fig. 1. The $R$ value estimated by MGI model is marked by the red arrow and with this $R$ value, the partial width of $\psi(3770) \rightarrow D \bar{D}$ is evaluated to be $29.1 \mathrm{MeV}$. The PDG average of the branching ratio for $\psi(3770) \rightarrow D \bar{D}$ is $\left(93_{-9}^{+8}\right) \%$ and the width of the $\psi(3770)$ is $27.2 \pm 1 \mathrm{MeV}$ [49]. Thus, the measured partial width of $\psi(3770) \rightarrow D \bar{D}$ is $22.7-$ $27.3 \mathrm{MeV}$, which indicates the partial width with $R$ value in MGI model is approximately consistent with the experimental measurement. Moreover, we vary $R$ value from $1.6 \mathrm{GeV}^{-1}$ to $2.0 \mathrm{GeV}^{-1}$ and find that the estimated partial width of $\psi(3770) \rightarrow D \bar{D}$ with $R=1.6 \sim 1.76 \mathrm{GeV}^{-1}$ could well reproduce the experimental measurement. Taking $\psi(3770)$ as a scale of $1 D$ charmonia, the partial width of $\psi_{3}(1 D) \rightarrow D \bar{D}$ is $0.34-0.46 \mathrm{MeV}$, which is consistent with the theoretical estimations in Refs. [50,51] and safely under the measured width from the LHCb Collaboration [31].

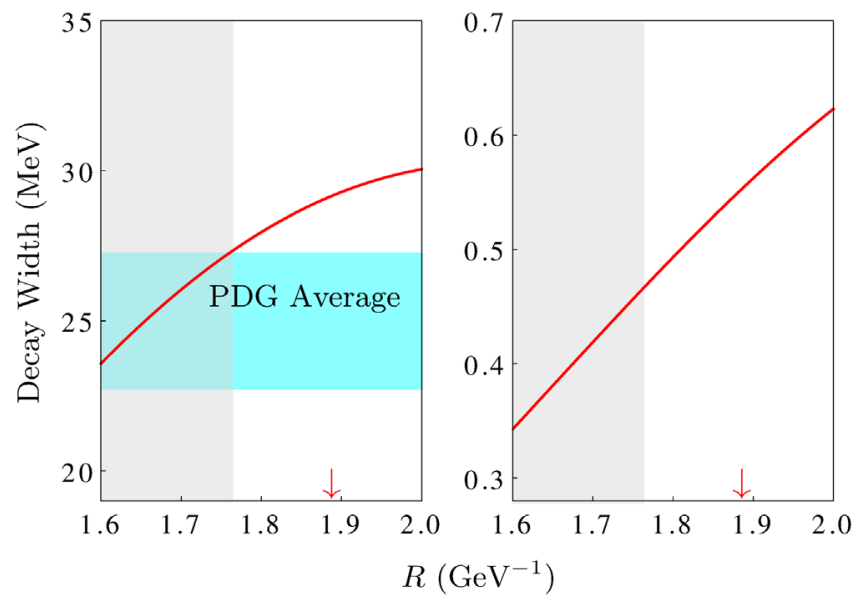

FIG. 1. The partial width of $\psi(1 D) \rightarrow D \bar{D}$ (left panel) and $\psi_{3}(1 D) \rightarrow D \bar{D}$ (right panel). The cyan band indicates the PDG average of the corresponding partial width. The $R$ value estimated by MGI model are marked by red arrow.

\section{B. Open charm decays of $2 D$ states}

The $R$ dependent partial and total widths of $\psi(4160)$ are presented in Fig. 2. By taking the $R$ value determined in MGI model, the width of $\psi(4160)$ is estimated to be 147.4 MeV, which is about two time larger than the PDG average one, i.e., $70 \pm 10 \mathrm{MeV}$. It should noticed that the mass of $\psi(2 D)$ is estimated to be $4.125 \mathrm{GeV}$ in the MGI model, which is much smaller than the measured one, thus we discuss the decay behavior of $2 D$ states in the $R$ range determined by comparing the estimated width with the experimental data [49], which is $R=(1.82-1.97) \mathrm{GeV}^{-1}$. Moreover, the determined $R$ value of $\psi(4160)$ is a bit larger than the one of $\psi(3770)$, which is consistent with the

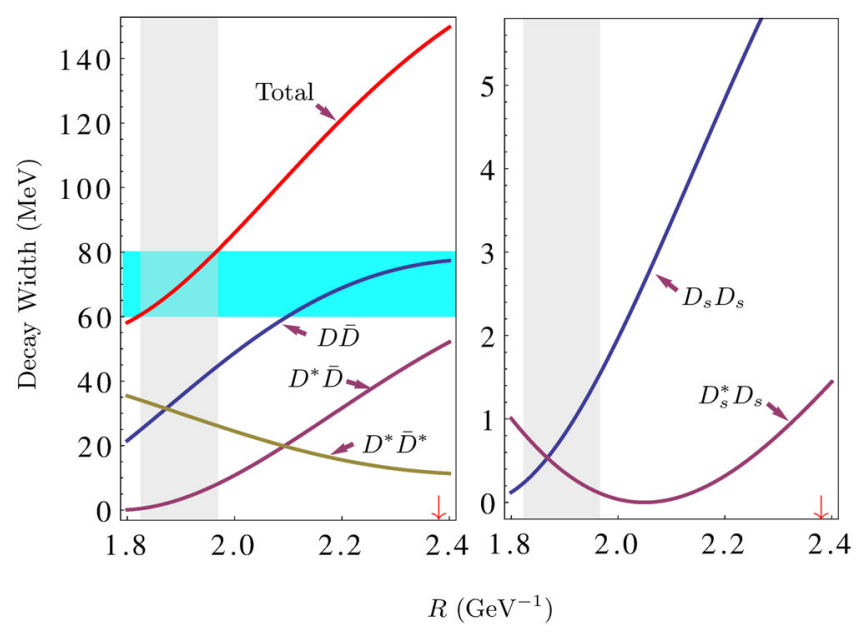

FIG. 2. Partial and total widths of $\psi(4160)$. The cyan horizontal band is the PDG average of the total width and the light vertical grey band is the $R$ range determined by the overlap of theoretical estimation and experimental data. The $R$ value estimated by MGI model are marked by red arrow. 
expectation. In this $R$ range, our results indicates the $\psi(4160)$ dominantly decays into $D \bar{D}, D^{*} \bar{D}$, and $D^{*} \bar{D}^{*}$, while the partial widths of $D_{s}^{+} D_{s}^{-}$and $D_{s}^{+} D_{s}^{*-}$ are less than $1 \mathrm{MeV}$. In this $R$ range, the ratios of the partial widths of open charmed processes are estimated to be

$$
\begin{aligned}
& \frac{\Gamma(\psi(4160) \rightarrow D \bar{D})}{\Gamma\left(\psi(4160) \rightarrow D^{*} \bar{D}^{*}\right)}=0.71-1.72 \\
& \frac{\Gamma\left(\psi(4160) \rightarrow D^{*} \bar{D}\right)}{\Gamma\left(\psi(4160) \rightarrow D^{*} \bar{D}^{*}\right)}=0.01-0.31
\end{aligned}
$$

These ratios are evaluated to be $0.46 / 0.01$ and $0.2 / 0.05$ by the QPC model with relativistic quark model and linear potential model, respectively [50,52]. In Ref. [53], by using the Connell coupled- channel mode, the ratios are determined to be 0.08 and 0.16 . On the experimental side, the $B A B A R$ collaboration performed a measurement of the exclusive production of $D \bar{D}, D^{*} \bar{D}$, and $D^{*} \bar{D}^{*}$, the ratios were measured to be $0.02 \pm 0.03 \pm 0.02$ and $0.34 \pm$ $0.14 \pm 0.05$ [54], respectively, which is different from the QPC model estimations in the present work. It should be noticed that in Ref. [54], the data are fitted with three charmonia with fixed mass and width, which are $\psi(4040)$, $\psi(4160)$, and $\psi(4415)$. From the current situation, there should exist more vector states in this energy range and thus the fitted results will be changed if more states are included. Moreover, in the analysis, the mass and width of $\psi(4160)$ are fixed to be $4153 \mathrm{MeV}$ and $103 \mathrm{MeV}$, respectively [55]. The values of the resonance parameters used in Ref. [54] are much different from latest PDG average, which are $4191 \mathrm{MeV}$ and $70 \mathrm{MeV}$, respectively [49]. We expect the new precise measurement and analysis of the open charm decays of $\psi(4160)$ at BESIII, BelleII, and LHCb could determine these ratios and test the results in the present work.

Taking the $R$ range determined by the width of $\psi(4160)$, we can investigate the open charm decays of other $2 D$ charmonium states. As for $\eta_{c 2}(2 D)$, the partial and total widths depending on $R$ value are presented in Fig. 3. The total width of $\eta_{c 2}(2 D)$ is estimated to be $48-64 \mathrm{MeV}$. The dominant decay modes are $D^{*} \bar{D}^{*}$ and $D^{*} \bar{D}$ and the ratio of these two decay channels is estimated to be

$$
\frac{\Gamma\left(\eta_{c 2}(2 D) \rightarrow D^{*} \bar{D}\right)}{\Gamma\left(\eta_{c 2}(2 D) \rightarrow D^{*} \bar{D}^{*}\right)}=0.7-1.5
$$

which indicates the partial width of $\Gamma\left(\eta_{c 2}(2 D) \rightarrow D^{*} \bar{D}\right)$ and $\Gamma\left(\eta_{c 2}(2 D) \rightarrow D^{*} \bar{D}^{*}\right)$ are very similar. As for the $D_{s}^{*+} D_{s}^{-}$and $D \bar{D}_{0}$ modes, their partial widths are less than $1 \mathrm{MeV}$.

As shown in Fig. 4, the total width of $\psi_{2}(2 D)$, is very weakly dependent on the $R$ values in the determined $R$ range, and it is estimated to be $50-52 \mathrm{MeV}$. Similar to the case of $\eta_{c 2}(2 D)$, the dominant decay modes of $\psi_{2}(2 D)$ are

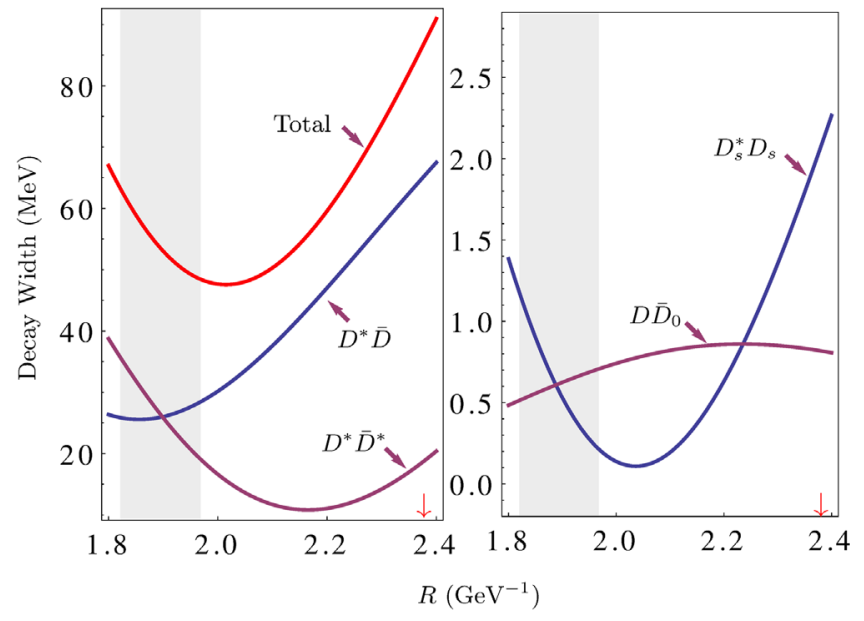

FIG. 3. Partial and total widths of $\eta_{c 2}(2 D)$. The light grey band is the $R$ range determined by the comparison of $\psi(4160)$ total width with the experimental data. The $R$ value estimated by MGI model are marked by the arrow.

also $D^{*} \bar{D}$ and $D^{*} \bar{D}^{*}$, and the ratio of the partial widths for these two channels are estimated to be,

$$
\frac{\Gamma\left(\psi_{2}(2 D) \rightarrow D^{*} \bar{D}\right)}{\Gamma\left(\psi_{2}(2 D) \rightarrow D^{*} \bar{D}^{*}\right)}=0.6-1.4
$$

As for $\psi_{3}(2 D)$, the total and partial widths are presented in Fig. 5. In the determined $R$ range, the total width is estimated to be 52-76 MeV. Such a large width mainly comes from the $D^{*} \bar{D}$ and $D^{*} \bar{D}^{*}$ modes since $\psi_{3}(2 D)$ decays into $D^{*} \bar{D}$ and $D^{*} \bar{D}^{*}$ are also via $P$ wave. The partial widths ratio of these two channel is predicted to be,

$$
\frac{\Gamma\left(\psi_{3}(2 D) \rightarrow D^{*} \bar{D}\right)}{\Gamma\left(\psi_{3}(2 D) \rightarrow D^{*} \bar{D}^{*}\right)}=0.5-0.8 .
$$

Compared with the above two channels, the partial width of $D \bar{D}$ mode is much smaller due to the high partial wave suppression.

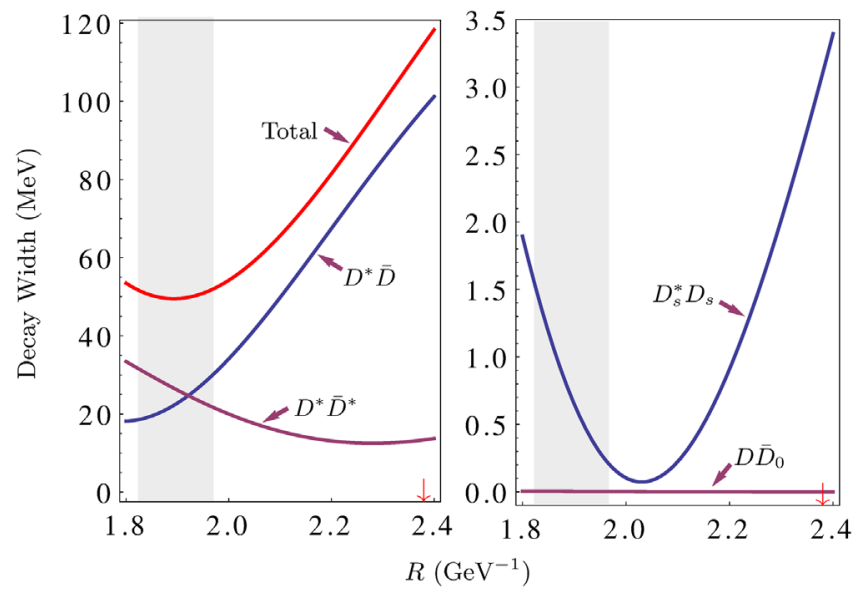

FIG. 4. The same as Fig. 3 but for $\psi_{2}(2 D)$ charmonium. 


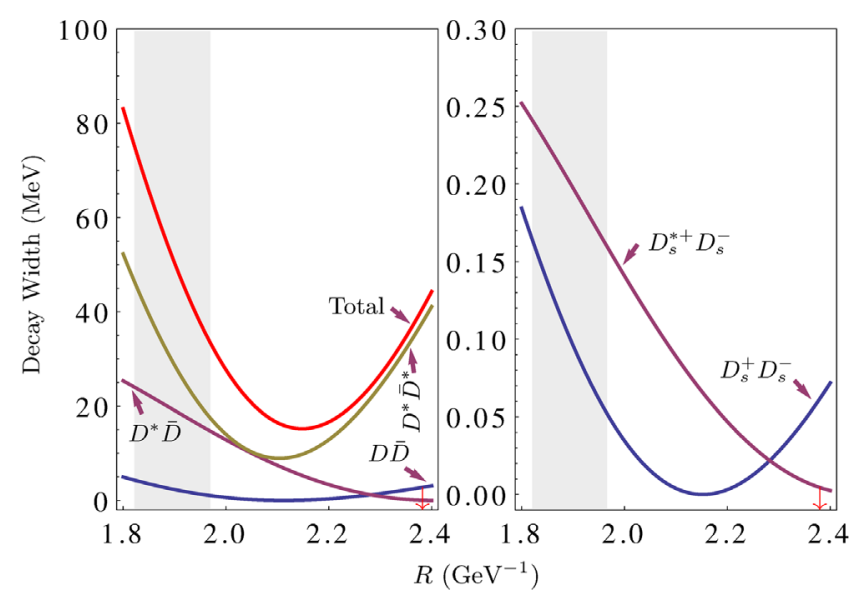

FIG. 5. The same as Fig. 3 but for $\psi_{3}(2 D)$ charmonium.

\section{Open charm decays of $3 D$ states}

The $R$ dependent total and partial widths of the open charm decays of $Y(4320)$ are presented in Fig. 6, where $Y(4320)$ is assigned as $\psi\left(3^{3} D_{1}\right)$ charmonium. In the MGI model, the $R$ value of $3 D$ charmonia is $2.85 \mathrm{GeV}^{-1}$ and with this $R$ value, the width of $\psi\left(3^{3} D_{1}\right)$ is $121.7 \mathrm{MeV}$, which is consistent with one of $Y(4320)$, i.e., $\left(101.4_{-19.7}^{+25.3} \pm\right.$ 10.2) $\mathrm{MeV}$ [2]. To further check the $R$ dependence of the total width and partial width of $\psi\left(3^{3} D_{1}\right)$, we vary $R$ value from $2.5 \mathrm{GeV}^{-1}$ to $3.0 \mathrm{GeV}^{-1}$. Our estimations indicate that when $R=2.50-2.92 \mathrm{GeV}^{-1}$ the evaluated total width are consistent with the measured one from the BESIII Collaboration [2]. This $R$ value for $\psi\left(3^{3} D_{1}\right)$ is larger than the one of $\psi(4160)$ and $\psi(3770)$, which is consistent with our expectation. In this $R$ range, $\psi\left(3^{3} D_{1}\right)$ dominantly decays into $D \bar{D}, D^{*} \bar{D}$, and $D^{*} \bar{D}^{*}$. The partial width of $\psi\left(3^{3} D_{1}\right) \rightarrow D \bar{D}$ weakly depend on the parameter $R$, and in the determined $R$ range, we find $\Gamma\left(\psi\left(3{ }^{3} D_{1}\right) \rightarrow D \bar{D}\right)=$ 45.2-48.0 MeV. And in this $R$ range, the partial widths of $D^{*} \bar{D}$ and $D^{*} \bar{D}^{*}$ are estimated to be 24.3-46.0 and

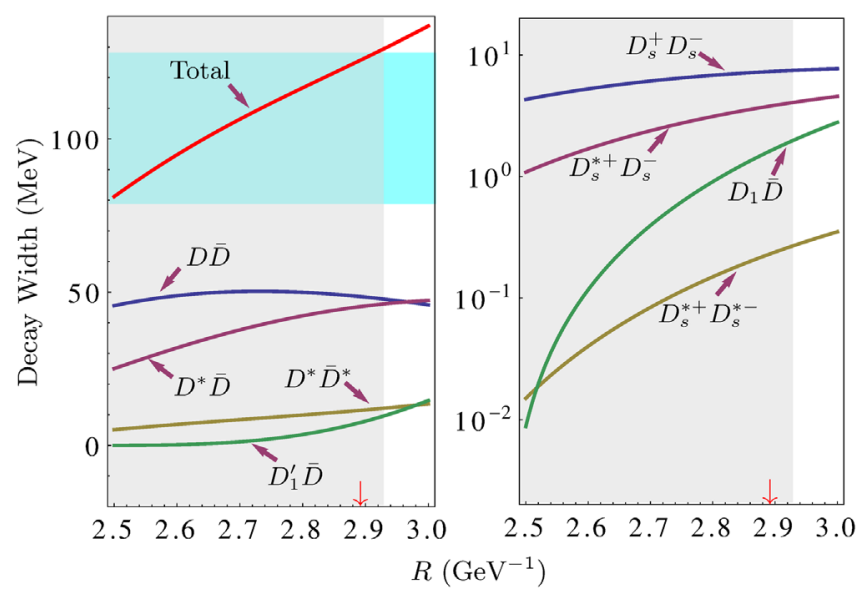

FIG. 6. The same as Fig. 2 but for $\psi_{1}(3 D)$.

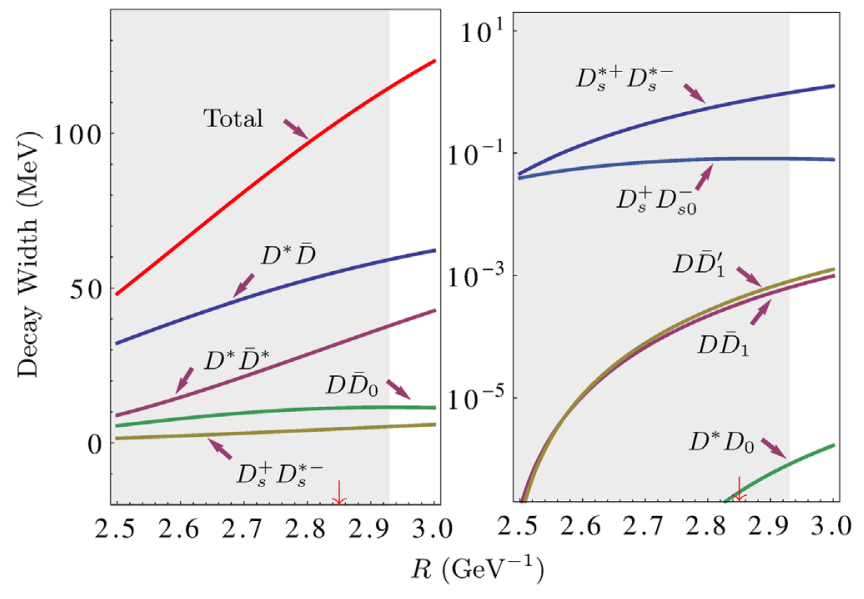

FIG. 7. Partial and total widths of $\eta_{c 2}(3 D)$. The light grey band is the $R$ range determined by the comparison of $Y(4320)$ total width with the experimental data, where $Y(4320)$ is assigned as $\psi_{1}(3 D)$ charmonium.

4.9-11.9 MeV, respectively. The ratios of the partial widths of these dominant decay channels are predicted to be

$$
\begin{aligned}
& \frac{\Gamma(Y(4320) \rightarrow D \bar{D})}{\Gamma\left(Y(4320) \rightarrow D^{*} \bar{D}^{*}\right)}=4.0-9.2 \\
& \frac{\Gamma\left(Y(4320) \rightarrow D^{*} \bar{D}\right)}{\Gamma\left(Y(4320) \rightarrow D^{*} \bar{D}^{*}\right)}=3.8-4.5
\end{aligned}
$$

respectively.

As for $\eta_{c 2}(3 D)$, the total and partial widths depending on the model parameter $R$ are presented in Fig. 7. In the $R$ range determined by $Y(4320)$, we find the total width of $\eta_{c 2}(3 D)$ is strongly dependent on the model parameter. In particular, the total width is estimated to be $47-114 \mathrm{MeV}$ in this $R$ range. Moreover, our estimation indicates that the $D^{*} \bar{D}$ and $D^{*} \bar{D}^{*}$ should be the dominant decay modes of $\eta_{c 2}(3 D)$ and the partial widths ratio of these two modes is estimated to be,

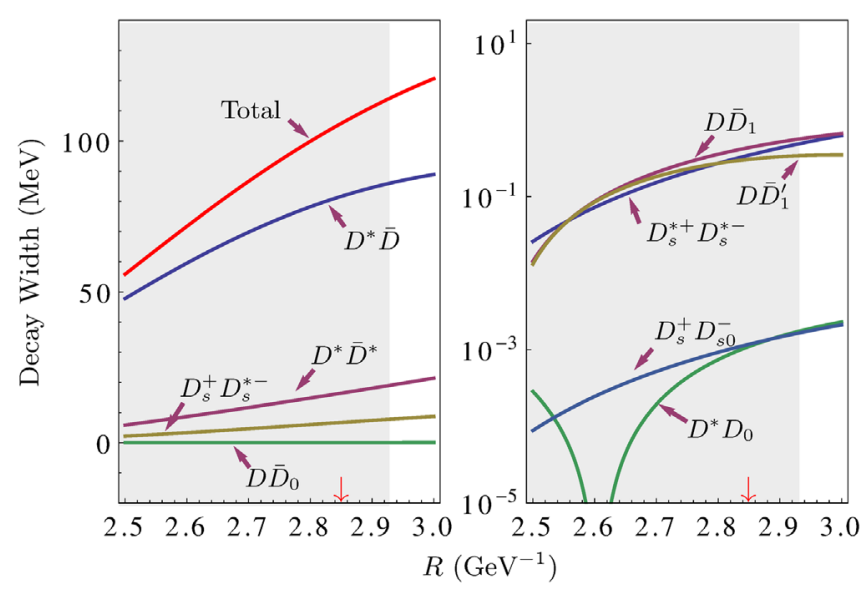

FIG. 8. The same as Fig. 7 but for $\psi_{2}(3 D)$ charmonium. 


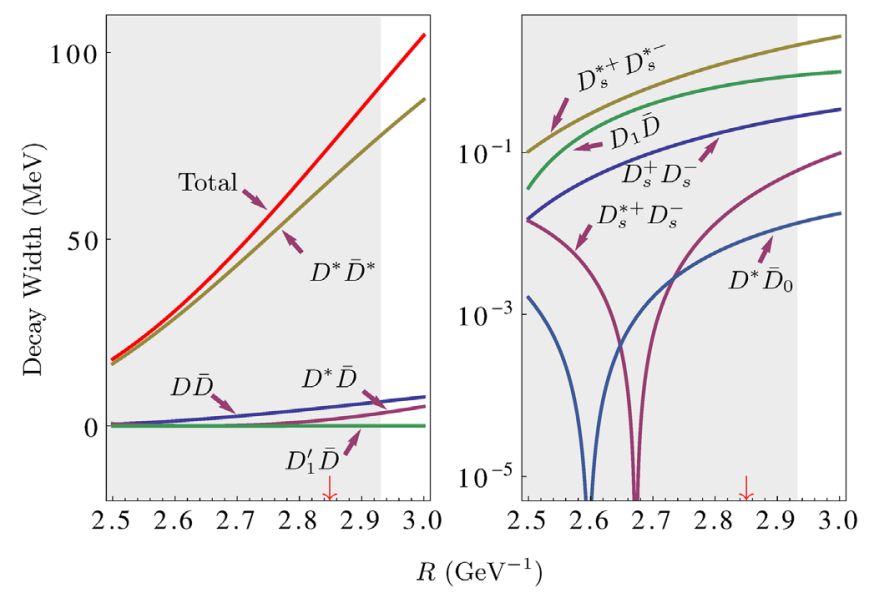

FIG. 9. The same as Fig. 7 but for $\psi_{3}(3 D)$ charmonium.

$$
\frac{\Gamma\left(\eta_{c 2} \rightarrow D^{*} \bar{D}\right)}{\Gamma\left(\eta_{c 2} \rightarrow D^{*} \bar{D}^{*}\right)}=1.6-3.8
$$

The total and partial widths of $\psi_{2}(3 D)$ are presented in Fig. 8 . The total width is estimated to be $54-113 \mathrm{MeV}$ and in the determined $R$ range, $D^{*} \bar{D}$ is the dominant decay modes, the branching ratio of this decay mode is (76-86)\%. As shown in Fig. 9, the total width of $\psi_{3}(3 D)$ is also strong dependent on the model parameter and predicted to be $17-89 \mathrm{MeV}$. In the determined $R$ range, the dominant decay mode is $D^{*} \bar{D}^{*}$, and its branching ratio is estimated to be (86-93)\%.

It should be noticed the measured width of $Y(4320)$ has a relative large uncertainty, thus in a large $R$ range our estimation could overlap with the measured data. Then the predicted total and partial widths for $\eta_{c 2}(3 D), \psi_{2}(3 D)$, and $\psi_{3}(3 D)$ vary in a relative large range. However, the dominant decay modes and the partial widths ratios are weakly dependent on the model parameter, which are helpful for searching these missing $3 D$ states.

\section{SUMMARY}

The observations of the vector charmoniumlike states in the $e^{+} e^{-}$annihilation processes make the states between 4.0 and $4.5 \mathrm{GeV}$ overcrowed. Besides the higher excited $J / \psi$ state, these charmoniumlike states could also be higher $\psi\left({ }^{3} D_{1}\right)$ states. Moreover, a $\psi_{3}(1 D)$ candidate was observed in the $D \bar{D}$ invariant mass spectroscopy very recently by the LHCb Collaboration. These experimental measurements stimulate us to comb $D$ wave charmonium states. In the present work, by investigating the open charm decay behaviors, we evaluate the possibility of $Y(4320)$ as $\psi\left(3^{3} D_{1}\right)$ charmonium and then take $\psi(3770), \psi(4160)$, and $Y(4320)$ as the scales to evaluate the open charm decays of other $1 D, 2 D$, and $3 D$ charmonium states.

Our estimations indicate that the total widths of $\psi(3770)$ and $\psi(4160)$ can be reproduced in a proper $R$ range, which are $R=1.60-1.76 \mathrm{GeV}^{-1}$ and $\left.1.82-1.97\right) \mathrm{GeV}^{-1}$ for $\psi(3770)$ and $\psi(4160)$, respectively. As for $Y(4320)$, the estimated total width can overlap with the measured one when we take $R=2.50-2.92 \mathrm{GeV}^{-1}$, which indicates $Y(4320)$ can be a $\psi_{1}(3 D)$ candidate. It should be notice that the $R$ range for $1 D$ is very close to the one of estimated in MGI model, and for $3 D$ charmonium, the $R$ value estimated in MGI model is consistent with the $R$ range determined by the width of $\psi\left(3^{3} D_{1}\right)$ state. However, as for $2 D$ state, the $R$ value estimated in MGI model is much larger than the range determined by the width of $\psi(4160)$.

Taking $\psi(3770), \psi(4160)$, and $\psi(4320)$ as the scale of $1 D, 2 D$, and $3 D$ charmonia, respectively, we can estimated the open charm decays of other $D$ wave charmonium states. From our estimations, we find,

(i) $\psi(4382)$ could be assigned as $\psi_{3}(1 D)$ state. The narrow width resulted from the high partial wave suppression and our estimated partial width $\psi_{3}(1 D) \rightarrow D \bar{D}$ is safely under the measured width of $\psi(3842)$ and consistent with the theoretical estimations of other group.

(ii) As for $2 D$ states, we predict the total widths of $\eta_{c 2}(2 D), \psi_{2}(2 D)$, and $\psi_{3}(2 D)$ to be $48-64,50-52$, and $52-76 \mathrm{MeV}$, respectively. We also find that the dominant decay modes of these three $D$ wave charmonia are $D^{*} \bar{D}$ and $D^{*} \bar{D}^{*}$, respectively. Furthermore, the partial widths ratios of these dominant channels are also predicted.

(iii) As for $3 D$ states, the predicted total widths of $\eta_{c 2}(3 D), \psi_{2}(3 D)$, and $\psi_{3}(3 D)$ are in a relative large range due to the large uncertainty of $R$ determined by the width of $Y(4320)$. However, we find the predominant decay modes are $D^{*} \bar{D}, D^{*} \bar{D}$, and $D^{*} \bar{D}^{*}$ for $\eta_{c 2}(3 D), \psi_{2}(3 D)$, and $\psi_{3}(3 D)$, respectively. Moreover, some partial widths ratios are predicted, which are nonsensitive to the model parameter $R$.

The open charm decay channels are the important observation channels of higher charmonia since they are the dominant decay channels of these higher charmonia. The charmonia produced in hadronproduction process have more possible $J^{P C}$ quantum numbers. Thus, hadronproduction is one of most promising process of searching for the higher $D$ wave charmonia in the open charm mass spectroscopy. All the estimations in the present work could be helpful for searching for the missing $2 D$ and $3 D$ charmonia in the open charm decay channels in the further experimental measurements at $\mathrm{LHCb}$.

\section{ACKNOWLEDGMENTS}

The authors would like to thank Jun-Zhang Wang for useful discussion. This project is supported by the National Natural Science Foundation of China under Grants No. 11775050, No. 11375240, No. 11675228, No. 11965016 and No. 11975075, Nature Science Foundation Projects of Qinghai Office of Science and Technology, No. 2017-ZJ-748, the Natural Science Foundation of Jiangsu Province of China under Contract No. BK20171349. 


\section{APPENDIX: PARTIAL WAVE AMPLITUDES}

TABLE IV. The partial wave amplitude of the open charm decays for ${ }^{1} D_{2}$ initial state. Here $\alpha=1 / \sqrt{3}$ is the factor resulted from the flavor matrix element and $I_{M_{L_{B}}, M_{L_{C}}}^{M_{L}, m}$ is defined in Eq. (4).

\begin{tabular}{ll}
\hline \hline Decay channel & Amplitude \\
\hline${ }^{1} D_{2} \rightarrow{ }^{1} S_{0}{ }^{3} S_{1}$ & $\mathcal{M}^{11}=\frac{2 \alpha}{5 \sqrt{3}} \sqrt{E_{A} E_{B} E_{C}} \gamma\left(I_{00}^{00}-\sqrt{3} I_{00}^{1-1}\right)$ \\
& $\mathcal{M}^{13}=\frac{\sqrt{2} \alpha}{15} \sqrt{E_{A} E_{B} E_{C} \gamma\left(-3 I_{00}^{00}-2 \sqrt{3} I_{00}^{1-1}\right)}$ \\
${ }^{1} D_{2} \rightarrow{ }^{1} S_{0}{ }^{3} S_{1}$ & $\mathcal{M}^{11}=\frac{2 \sqrt{2} \alpha}{15} \sqrt{E_{A} E_{B} E_{C}} \gamma\left(\sqrt{3} I_{00}^{00}-3 I_{00}^{1-1}\right)$ \\
& $\mathcal{M}^{13}=-\frac{\sqrt{2} \alpha}{15} \sqrt{E_{A} E_{B} E_{C}} \gamma\left(3 I_{00}^{00}+2 \sqrt{3} I_{00}^{1-1}\right)$ \\
${ }^{1} D_{2} \rightarrow{ }^{1} S_{0}{ }^{3} P_{0}$ & $\mathcal{M}^{02}=-\frac{\sqrt{2} \alpha}{3 \sqrt{5}} \sqrt{E_{A} E_{B} E_{C}} \gamma\left(I_{00}^{00}+2 I_{01}^{01}\right)$ \\
${ }^{1} D_{2} \rightarrow{ }^{1} S_{0} P_{1}$ & $\mathcal{M}^{12}=\cos \theta\left[-\sqrt{\frac{2}{15}} \alpha \sqrt{E_{A} E_{B} E_{C}} \gamma\left(I_{00}^{1-1}+2 I_{01}^{10}\right)\right]$ \\
${ }^{1} D_{2} \rightarrow{ }^{1} S_{0} P_{1}^{\prime}$ & $\mathcal{M}^{12}=\sin \theta\left[-\sqrt{\frac{2}{15}} \alpha \sqrt{E_{A} E_{B} E_{C}} \gamma\left(I_{00}^{1-1}+2 I_{01}^{10}\right)\right]$ \\
${ }^{1} D_{2} \rightarrow{ }^{3} S_{1}{ }^{3} P_{0}$ & $\mathcal{M}^{12}=-\frac{2 \alpha}{3 \sqrt{5}} \sqrt{E_{A} E_{B} E_{C}} \gamma\left(I_{00}^{1-1}+2 I_{01}^{10}\right)$ \\
\hline \hline
\end{tabular}

TABLE V. The same as Table. IV but for ${ }^{3} D_{1}$ initial state.

\begin{tabular}{|c|c|}
\hline Decay channel & Amplitude \\
\hline${ }^{3} D_{1} \rightarrow{ }^{1} S_{0}{ }^{1} S_{0}$ & $\mathcal{M}^{01}=\frac{2 \alpha}{3 \sqrt{5}} \sqrt{E_{A} E_{B} E_{C}} \gamma\left(\sqrt{3} I_{00}^{1-1}-I_{00}^{00}\right)$ \\
\hline${ }^{3} D_{1} \rightarrow{ }^{3} S_{1}{ }^{1} S_{0}$ & $\mathcal{M}^{11}=\frac{2 \alpha}{\sqrt{30}} \sqrt{E_{A} E_{B} E_{C}} \gamma\left(\sqrt{3} I_{00}^{00}-3 I_{00}^{1-1}\right)$ \\
\hline${ }^{3} D_{1} \rightarrow{ }^{3} S_{1}{ }^{3} S_{1}$ & $\begin{array}{l}\mathcal{M}^{01}=\frac{2 \alpha}{3 \sqrt{15}} \sqrt{E_{A} E_{B} E_{C}} \gamma\left(I_{00}^{00}-\sqrt{3} I_{00}^{1-1}\right) \\
\mathcal{M}^{21}=\frac{2 \alpha}{45} \sqrt{E_{A} E_{B} E_{C}} \gamma\left(3 I_{00}^{1-1}-\sqrt{3} I_{00}^{00}\right) \\
\mathcal{M}^{23}=\frac{2 \sqrt{2} \alpha}{\sqrt{45}} \sqrt{E_{A} E_{B} E_{C}} \gamma\left(3 I_{00}^{00}+2 \sqrt{3} I_{00}^{1-1}\right)\end{array}$ \\
\hline${ }^{3} D_{1} \rightarrow{ }^{1} S_{0} P_{1}^{\prime}$ & $\begin{aligned} \mathcal{M}^{10}= & \cos \theta\left[\frac{2 \alpha}{9 \sqrt{5}} \sqrt{E_{A} E_{B} E_{C}} \gamma\left(-\sqrt{3} I_{00}^{00}+\sqrt{3} I_{01}^{01}+3 I_{00}^{1-1}-3 I_{01}^{10}+3 \sqrt{2} I_{01}^{2-1}\right)\right] \\
& +\sin \theta\left[\frac{\alpha}{9} \sqrt{\frac{2}{5}} \sqrt{E_{A} E_{B} E_{C}} \gamma\left(\sqrt{3} I_{00}^{00}-\sqrt{3} I_{01}^{01}-3 I_{00}^{1-1}+3 I_{01}^{10}-3 \sqrt{2} I_{01}^{2-1}\right)\right] \\
\mathcal{M}^{12}= & \cos \theta\left[\frac{\alpha}{9 \sqrt{5}} \sqrt{E_{A} E_{B} E_{C}} \gamma\left(2 \sqrt{6} I_{00}^{00}+\sqrt{6} I_{01}^{01}-6 \sqrt{2} I_{00}^{1-1}-3 \sqrt{2} I_{01}^{10}+6 I_{01}^{2-1}\right)\right] \\
& +\sin \theta\left[\frac{\alpha}{9 \sqrt{5}} \sqrt{E_{A} E_{B} E_{C}} \gamma\left(\sqrt{3} I_{00}^{00}+5 \sqrt{3} I_{01}^{01}-3 \sqrt{2} I_{00}^{1-1}-6 \sqrt{2} I_{01}^{10}-3 \sqrt{2} I_{01}^{2-1}\right)\right]\end{aligned}$ \\
\hline${ }^{3} D_{1} \rightarrow{ }^{1} S_{0} P_{1}$ & $\begin{aligned} \mathcal{M}^{10}= & -\sin \theta\left[\frac{2 \alpha}{9 \sqrt{5}} \sqrt{E_{A} E_{B} E_{C}} \gamma\left(-\sqrt{3} I_{00}^{00}+\sqrt{3} I_{01}^{01}+3 I_{00}^{1-1}-3 I_{01}^{10}+3 \sqrt{2} I_{01}^{2-1}\right)\right] \\
& +\cos \theta\left[\frac{\alpha}{9} \sqrt{\frac{2}{5}} \sqrt{E_{A} E_{B} E_{C}} \gamma\left(\sqrt{3} I_{00}^{00}-\sqrt{3} I_{01}^{01}-3 I_{00}^{1-1}+3 I_{01}^{10}-3 \sqrt{2} I_{01}^{2-1}\right)\right] \\
\mathcal{M}^{12}= & -\sin \theta\left[\frac{\alpha}{9 \sqrt{5}} \sqrt{E_{A} E_{B} E_{C}} \gamma\left(2 \sqrt{6} I_{00}^{00}+\sqrt{6} I_{01}^{01}-6 \sqrt{2} I_{00}^{1-1}-3 \sqrt{2} I_{01}^{10}+6 I_{01}^{2-1}\right)\right] \\
& +\cos \theta\left[\frac{\alpha}{9 \sqrt{5}} \sqrt{E_{A} E_{B} E_{C}} \gamma\left(\sqrt{3} I_{00}^{00}+5 \sqrt{3} I_{01}^{01}-3 \sqrt{2} I_{00}^{1-1}-6 \sqrt{2} I_{01}^{10}-3 \sqrt{2} I_{01}^{2-1}\right)\right]\end{aligned}$ \\
\hline${ }^{3} D_{1} \rightarrow{ }^{1} S_{0}{ }^{3} P_{2}$ & $\mathcal{M}^{22}=\frac{\alpha}{3 \sqrt{5}} \sqrt{E_{A} E_{B} E_{C}} \gamma\left(-I_{00}^{00}+I_{01}^{01}+\sqrt{3} I_{00}^{1-1}-\sqrt{6} I_{01}^{2-1}\right)$ \\
\hline${ }^{3} D_{1} \rightarrow{ }^{3} S_{1}{ }^{3} P_{0}$ & $\mathcal{M}^{1,2}=\frac{\alpha}{3} \sqrt{\frac{2}{5}} \sqrt{E_{A} E_{B} E_{C}} \gamma\left(I_{00}^{00}+2 I_{01}^{01}+\sqrt{3} I_{00}^{1-1}+I_{01}^{10}\right)$ \\
\hline
\end{tabular}


TABLE VI. The same as Table IV but for ${ }^{3} D_{2}$ initial state.

\begin{tabular}{|c|c|}
\hline Decay channel & Amplitude \\
\hline \multirow[t]{2}{*}{${ }^{3} D_{2} \rightarrow{ }^{1} S_{0}{ }^{3} S_{1}$} & $\mathcal{M}^{11}=\frac{\sqrt{2} \alpha}{5} \sqrt{E_{A} E_{B} E_{C}} \gamma\left(I_{00}^{00}-\sqrt{3} I_{00}^{1-1}\right)$ \\
\hline & $\mathcal{M}^{13}=\frac{2 \alpha}{15} \sqrt{E_{A} E_{B} E_{C}} \gamma\left(\sqrt{3} I_{00}^{00}+2 I_{00}^{1-1}\right)$ \\
\hline \multirow[t]{2}{*}{${ }^{3} D_{2} \rightarrow{ }^{3} S_{1}{ }^{3} S_{1}$} & $\mathcal{M}^{21}=\frac{\sqrt{2} \alpha}{15} \sqrt{E_{A} E_{B} E_{C}} \gamma\left(3 \sqrt{2} I_{00}^{1-1}-\sqrt{6} I_{00}^{00}\right)$ \\
\hline & $\mathcal{M}^{23}=\frac{4 \alpha}{15} \sqrt{E_{A} E_{B} E_{C}} \gamma\left(\sqrt{3} I_{00}^{00}+2 I_{00}^{1-1}\right)$ \\
\hline${ }^{3} D_{2} \rightarrow{ }^{1} S_{0}{ }^{3} P_{0}$ & $\mathcal{M}^{02}=\frac{2 \alpha}{3 \sqrt{5}} \sqrt{E_{A} E_{B} E_{C}} \gamma\left(\sqrt{3} I_{00}^{1-1}+2 I_{00}^{1-1}\right)$ \\
\hline${ }^{3} D_{2} \rightarrow{ }^{1} S_{0}{ }^{3} P_{0}$ & $\mathcal{M}^{02}=\frac{2 \alpha}{3 \sqrt{5}} \sqrt{E_{A} E_{B} E_{C}} \gamma\left(\sqrt{3} I_{00}^{1-1}+2 I_{00}^{1-1}\right)$ \\
\hline${ }^{3} D_{2} \rightarrow{ }^{1} S_{0} P_{1}$ & $\begin{aligned} \mathcal{M}^{12}= & -\sin \theta\left[\frac{\sqrt{2} \alpha}{3 \sqrt{5}} \sqrt{E_{A} E_{B} E_{C}} \gamma\left(\sqrt{3} I_{01}^{01}-I_{01}^{10}-\sqrt{2} I_{01}^{2-1}\right)\right] \\
& +\cos \theta\left[\frac{\alpha}{3 \sqrt{5}} \sqrt{E_{A} E_{B} E_{C}} \gamma\left(\sqrt{3} I_{00}^{00}+\sqrt{3} I_{01}^{01}-I_{00}^{1-1}+\sqrt{2} I_{01}^{2-1}\right)\right]\end{aligned}$ \\
\hline${ }^{3} D_{2} \rightarrow{ }^{1} S_{0} P_{1}^{\prime}$ & $\mathcal{M}^{12}=\cos \theta\left[\frac{\sqrt{2} \alpha}{3 \sqrt{5}} \sqrt{E_{A} E_{B} E_{C}} \gamma\left(\sqrt{3} I_{01}^{01}-I_{01}^{10}-\sqrt{2} I_{01}^{2-1}\right)\right]$ \\
\hline & $+\sin \theta\left[\frac{\alpha}{3 \sqrt{5}} \sqrt{E_{A} E_{B} E_{C}} \gamma\left(\sqrt{3} I_{00}^{00}+\sqrt{3} I_{01}^{01}-I_{00}^{1-1}+\sqrt{2} I_{01}^{2-1}\right)\right]$ \\
\hline${ }^{\mathrm{D}} \mathrm{D}_{2} \rightarrow{ }^{\mathrm{J}} S_{1}{ }^{\mathrm{S}} P_{0}$ & $\mathcal{M}^{12}=\frac{\sqrt{2} \alpha}{9 \sqrt{5}} \sqrt{E_{A} E_{B} E_{C}} \gamma\left(3 I_{00}^{00}+6 I_{01}^{01}+\sqrt{3} I_{00}^{1-1}+I_{01}^{10}\right)$ \\
\hline
\end{tabular}

TABLE VII. The same as Table IV but for ${ }^{3} D_{3}$ initial state.

\begin{tabular}{|c|c|}
\hline Decay channel & Amplitude \\
\hline${ }^{3} D_{3} \rightarrow{ }^{1} S_{0}{ }^{1} S_{0}$ & $\mathcal{M}^{03}=\frac{\sqrt{2} \alpha}{3 \sqrt{35}} \sqrt{E_{A} E_{B} E_{C}} \gamma\left(3 I_{00}^{00}+2 \sqrt{3} I_{00}^{1-1}\right)$ \\
\hline${ }^{3} D_{3} \rightarrow{ }^{1} S_{0}{ }^{3} S_{1}$ & $\mathcal{M}^{13}=\frac{2 \sqrt{2} \alpha}{3 \sqrt{35}} \sqrt{E_{A} E_{B} E_{C}} \gamma\left(\sqrt{3} I_{00}^{00}+2 I_{00}^{1-1}\right)$ \\
\hline \multirow[t]{3}{*}{${ }^{3} D_{3} \rightarrow{ }^{3} S_{1}{ }^{3} S_{1}$} & $\mathcal{M}^{03}=-\frac{\sqrt{2} \alpha}{3 \sqrt{35}} \sqrt{E_{A} E_{B} E_{C}} \gamma\left(\sqrt{3} I_{00}^{00}+2 I_{00}^{1-1}\right)$ \\
\hline & $\mathcal{M}^{21}=\frac{2 \sqrt{2} \alpha}{15} \sqrt{E_{A} E_{B} E_{C}} \gamma\left(3 \sqrt{2} I_{00}^{1-1}-\sqrt{6} I_{00}^{00}\right)$ \\
\hline & $\mathcal{M}^{23}=\frac{4 \alpha}{15 \sqrt{7}} \sqrt{E_{A} E_{B} E_{C}} \gamma\left(3 I_{00}^{00}+2 \sqrt{3} I_{00}^{1-1}\right)$ \\
\hline \multirow[t]{2}{*}{${ }^{3} D_{3} \rightarrow{ }^{1} S_{0} P_{1}$} & $\begin{aligned} \mathcal{M}^{12}= & -\sin \theta\left[\frac{\sqrt{2} \alpha}{21 \sqrt{5}} \sqrt{E_{A} E_{B} E_{C}} \gamma\left(3 \sqrt{3} I_{00}^{00}+4 \sqrt{3} I_{01}^{01}+6 I_{00}^{1-1}+8 I_{01}^{10}+2 \sqrt{2} I_{01}^{2-1}\right)\right] \\
& \cos \theta\left[\frac{\sqrt{2} \alpha}{21 \sqrt{5}} \sqrt{E_{A} E_{B} E_{C}} \gamma\left(2 \sqrt{6} I_{00}^{00}+5 \sqrt{6} I_{01}^{01}+4 \sqrt{2} I_{00}^{1-1}+3 \sqrt{2} I_{01}^{10}-2 I_{01}^{2-1}\right)\right]\end{aligned}$ \\
\hline & $\begin{aligned} \mathcal{M}^{14}= & -\sin \theta\left[\frac{\sqrt{2} \alpha}{21 \sqrt{5}} \sqrt{E_{A} E_{B} E_{C}} \gamma\left(-6 I_{00}^{00}+6 I_{01}^{01}-4 \sqrt{3} I_{00}^{1-1}+4 \sqrt{3} I_{01}^{10}+\sqrt{6} I_{01}^{2-1}\right)\right] \\
& \cos \theta\left[\frac{\sqrt{2} \alpha}{105} \sqrt{E_{A} E_{B} E_{C}} \gamma\left(3 \sqrt{10} I_{00}^{00}-3 \sqrt{10} I_{01}^{01}+2 \sqrt{30} I_{00}^{1-1}-2 \sqrt{30} I_{01}^{10}-\sqrt{15} I_{01}^{2-1}\right)\right.\end{aligned}$ \\
\hline \multirow[t]{2}{*}{${ }^{3} D_{3} \rightarrow{ }^{1} S_{0} P_{1}^{\prime}$} & $\begin{aligned} \mathcal{M}^{12}= & \cos \theta\left[\frac{\sqrt{2} \alpha}{21 \sqrt{5}} \sqrt{E_{A} E_{B} E_{C}} \gamma\left(3 \sqrt{3} I_{00}^{00}+4 \sqrt{3} I_{01}^{01}+6 I_{00}^{1-1}+8 I_{01}^{10}+2 \sqrt{2} I_{01}^{2-1}\right)\right] \\
& \sin \theta\left[\frac{\sqrt{2} \alpha}{21 \sqrt{5}} \sqrt{E_{A} E_{B} E_{C}} \gamma\left(2 \sqrt{6} I_{00}^{00}+5 \sqrt{6} I_{01}^{11}+4 \sqrt{2} I_{00}^{1-1}+3 \sqrt{2} I_{01}^{10}-2 I_{01}^{2-1}\right)\right]\end{aligned}$ \\
\hline & $\begin{aligned} \mathcal{M}^{14}= & \cos \theta\left[\frac{\sqrt{2} \alpha}{21 \sqrt{5}} \sqrt{E_{A} E_{B} E_{C}} \gamma\left(-6 I_{00}^{00}+6 I_{01}^{01}-4 \sqrt{3} I_{00}^{1-1}+4 \sqrt{3} I_{01}^{10}+\sqrt{6} I_{01}^{2-1}\right)\right] \\
& \sin \theta\left[\frac{\sqrt{2} \alpha}{105} \sqrt{E_{A} E_{B} E_{C}} \gamma\left(3 \sqrt{10} I_{00}^{00}-3 \sqrt{10} I_{01}^{01}+2 \sqrt{30} I_{00}^{1-1}-2 \sqrt{30} I_{01}^{10}-\sqrt{15} I_{01}^{2-1}\right)\right]\end{aligned}$ \\
\hline \multirow[t]{2}{*}{${ }^{3} D_{3} \rightarrow{ }^{1} S_{0}{ }^{3} P_{2}$} & $\mathcal{M}^{22}=\frac{2 \sqrt{2} \alpha}{21 \sqrt{5}} \sqrt{E_{A} E_{B} E_{C}} \gamma\left(\sqrt{3} I_{00}^{00}-\sqrt{3} I_{01}^{01}+2 I_{00}^{1-1}+5 I_{01}^{10}+3 \sqrt{2} I_{01}^{2-1}\right)$ \\
\hline & $\mathcal{M}^{24}=\frac{\sqrt{2} \alpha}{21} \sqrt{E_{A} E_{B} E_{C}} \gamma\left(-\sqrt{6} I_{00}^{00}+\sqrt{6} I_{01}^{01}-2 \sqrt{2} I_{00}^{1-1}+2 \sqrt{2} I_{01}^{10}+I_{01}^{2-1}\right)$ \\
\hline${ }^{3} D_{3} \rightarrow{ }^{3} S_{1}{ }^{3} P_{0}$ & $\mathcal{M}^{12}=\frac{\sqrt{2} \alpha}{9 \sqrt{5}} \sqrt{E_{A} E_{B} E_{C}} \gamma\left(3 I_{00}^{00}+6 I_{01}^{01}+2 I_{00}^{1-1}-2 \sqrt{3} I_{00}^{1-1}+I_{01}^{10}\right)$ \\
\hline
\end{tabular}


[1] M. Ablikim et al. (BESIII Collaboration), Phys. Rev. Lett. 114, 092003 (2015).

[2] M. Ablikim et al. (BESIII Collaboration), Phys. Rev. Lett. 118, 092001 (2017).

[3] M. Ablikim et al. (BESIII Collaboration), Phys. Rev. Lett. 118, 092002 (2017).

[4] Chang-Zheng Yuan for the BESIII Collaboration, $e^{+} e^{-}$ annihilation cross section measurements at BESIII, 10th workshop of the France China Particle Physics Laboratory (Tsinghua University, Beijing, China, 2017), http://indico .ihep.ac.cn/event/6651/timetableV\#20170329.detailed.

[5] B. Aubert et al. (BABAR Collaboration), Phys. Rev. Lett. 95, 142001 (2005).

[6] C. Z. Yuan et al. (Belle Collaboration), Phys. Rev. Lett. 99, 182004 (2007).

[7] G. J. Ding, Phys. Rev. D 79, 014001 (2009).

[8] M. Cleven, Q. Wang, F. K. Guo, C. Hanhart, U. G. Meißner, and Q. Zhao, Phys. Rev. D 90, 074039 (2014).

[9] M. Cleven and Q. Zhao, Phys. Lett. B 768, 52 (2017).

[10] S. R. Xue, H. J. Jing, F. K. Guo, and Q. Zhao, Phys. Lett. B 779, 402 (2018).

[11] D. Y. Chen, Y. B. Dong, M. T. Li, and W. L. Wang, Eur. Phys. J. A 52, 310 (2016).

[12] Y. Dong, A. Faessler, T. Gutsche, and V. E. Lyubovitskij, Phys. Rev. D 89, 034018 (2014).

[13] J. M. Dias, R. M. Albuquerque, M. Nielsen, and C. M. Zanetti, Phys. Rev. D 86, 116012 (2012).

[14] Z. G. Wang, Eur. Phys. J. C 76, 387 (2016).

[15] L. P. He, D. Y. Chen, X. Liu, and T. Matsuki, Eur. Phys. J. C 74, 3208 (2014).

[16] D. Y. Chen, X. Liu, and T. Matsuki, Phys. Rev. D 91, 094023 (2015).

[17] D. Y. Chen, X. Liu, and T. Matsuki, Phys. Rev. D 93, 034028 (2016).

[18] J. He and D. Y. Chen, Eur. Phys. J. C 77, 398 (2017).

[19] D. Y. Chen, C. J. Xiao, and J. He, Phys. Rev. D 96, 054017 (2017).

[20] D. Y. Chen, X. Liu, and T. Matsuki, Eur. Phys. J. C 78, 136 (2018).

[21] B. Aubert et al. (BABAR Collaboration), Phys. Rev. Lett. 98, 212001 (2007).

[22] X. L. Wang et al. (Belle Collaboration), Phys. Rev. Lett. 99, 142002 (2007).

[23] J. Zhang and L. Yuan, Eur. Phys. J. C 77, 727 (2017).

[24] S. Godfrey and N. Isgur, Phys. Rev. D 32, 189 (1985).

[25] B. Q. Li, C. Meng, and K. T. Chao, Phys. Rev. D 80, 014012 (2009).

[26] Y. S. Kalashnikova, Phys. Rev. D 72, 034010 (2005).

[27] P. G. Ortega, J. Segovia, D. R. Entem, and F. Fernandez, Phys. Rev. D 81, 054023 (2010).

[28] B. Q. Li and K. T. Chao, Phys. Rev. D 79, 094004 (2009).

[29] J. Z. Wang, D. Y. Chen, X. Liu, and T. Matsuki, Phys. Rev. D 99, 114003 (2019).
[30] M. Tanabashi et al. (Particle Data Group), Phys. Rev. D 98, 030001 (2018).

[31] T. Skwarnicki (on behalf of the LHCb Collaboration), http:// moriond.in2p3.fr/QCD/2019/TuesdayMorning/Skwarnicki .pptx.

[32] V. Bhardwaj et al. (Belle Collaboration), Phys. Rev. Lett. 111, 032001 (2013).

[33] M. Ablikim et al. (BESIII Collaboration), Phys. Rev. Lett. 115, 011803 (2015).

[34] L. Micu, Nucl. Phys. B10, 521 (1969).

[35] A. Le Yaouanc, L. Oliver, O. Pene, and J. C. Raynal, Phys. Rev. D 8, 2223 (1973).

[36] A. Le Yaouanc, L. Oliver, O. Pene, and J.-C. Raynal, Phys. Rev. D 9, 1415 (1974).

[37] A. Le Yaouanc, L. Oliver, O. Pene, and J.-C. Raynal, Phys. Lett. 71B, 397 (1977).

[38] X. Liu, Z. G. Luo, and Z. F. Sun, Phys. Rev. Lett. 104, 122001 (2010).

[39] D. Y. Chen, Eur. Phys. J. C 76, 671 (2016).

[40] Q. T. Song, D. Y. Chen, X. Liu, and T. Matsuki, Phys. Rev. D 92, 074011 (2015).

[41] Q. T. Song, D. Y. Chen, X. Liu, and T. Matsuki, Phys. Rev. D 91, 054031 (2015).

[42] Q. T. Song, D. Y. Chen, X. Liu, and T. Matsuki, Eur. Phys. J. C 75, 30 (2015).

[43] S. Godfrey and K. Moats, Phys. Rev. D 92, 054034 (2015).

[44] F. E. Close and E. S. Swanson, Phys. Rev. D 72, 094004 (2005).

[45] T. Barnes and S. Godfrey, Phys. Rev. D 69, 054008 (2004).

[46] S. Godfrey and R. Kokoski, Phys. Rev. D 43, 1679 (1991).

[47] T. Barnes, N. Black, and P. R. Page, Phys. Rev. D 68, 054014 (2003).

[48] T. Matsuki, T. Morii, and K. Seo, Prog. Theor. Phys. 124, 285 (2010).

[49] C. Patrignani et al. (Particle Data Group), Chin. Phys. C 40, 100001 (2016).

[50] T. Barnes, S. Godfrey, and E. S. Swanson, Phys. Rev. D 72, 054026 (2005).

[51] W. J. Deng, H. Liu, L. C. Gui, and X. H. Zhong, Phys. Rev. D 95, 034026 (2017).

[52] L. C. Gui, L. S. Lu, Q. F. Lü, X. H. Zhong, and Q. Zhao, Phys. Rev. D 98, 016010 (2018).

[53] E. J. Eichten, K. Lane, and C. Quigg, Phys. Rev. D 73, 014014 (2006); 73, 079903(E) (2006).

[54] B. Aubert et al. (BABAR Collaboration), Phys. Rev. D 79, 092001 (2009).

[55] C. Amsler et al. (Particle Data Group), Phys. Lett. B 667, 1 (2008). 